\title{
Im Fallbeispiel: Wiedererlangen der Alltagskompetenzen nach einem Sturzereignis
}

\author{
Sturz und seine Folgen In diesem Fallbeispiel wird auf Grundlage der Anamnese und \\ der ausgewählten Assessments die Auswahl von Pflegekonzepten zum Thema \\ Mobilität und Sturz erläutert. Wie kann durch die Umsetzung des Pflegeprozesses das \\ individuelle Ziel optimal erreichbar gemacht werden?
}

Das Ziel von Herrn N. war die Rückkehr in die vertraute häusliche Umgebung (Symbolbild).
- René Thiel -

Aktuelle Anamnese

Ein 82-jähriger Patient, Herr N., stolperte in der häuslichen Umgebung beim Hinabgehen einer Treppe und stürzte drei Stufen abwärts. Mit Hilfe des Notfallknopfes konnte der RTW alarmiert werden. Es folgte ein stationärer Aufenthalt, im Rahmen dessen eine Totalendoprothese des Hüftgelenks rechts bei einem unkomplizierten postoperativen Verlauf implantiert wurde. Um die Mobilität und Selbstständigkeit wiederzuerlangen, wurde der Patient zur frührehabilitativen Komplexbehandlung in der Geriatrie aufgenommen. Beim Aufnahmegespräch lässt sich eine reduzierte Konzentrationsfähigkeit feststellen.

Relevante Vorerkrankungen

Rezidivierende Stürze, Polyneuropathie bei Diabetes mellitus Typ 2, arterieller Hypertonus, Herzinsuffizienz, Unterschenkelödeme, beidseitig operierter Grauer Star.

\section{Sozialanamnese}

Herr N. lebt allein in einem Einfamilienhaus, dort hat er 10 Treppenstufen zu bewältigen. Ein Pflegegrad liegt nicht vor. Seine Ehefrau verstarb 2001. Sein Sohn, der $150 \mathrm{~km}$ entfernt wohnt, hat die Vorsorgegeneralvollmacht. Eine Patientenverfügung liegt bereits vor. Der Patient wird 


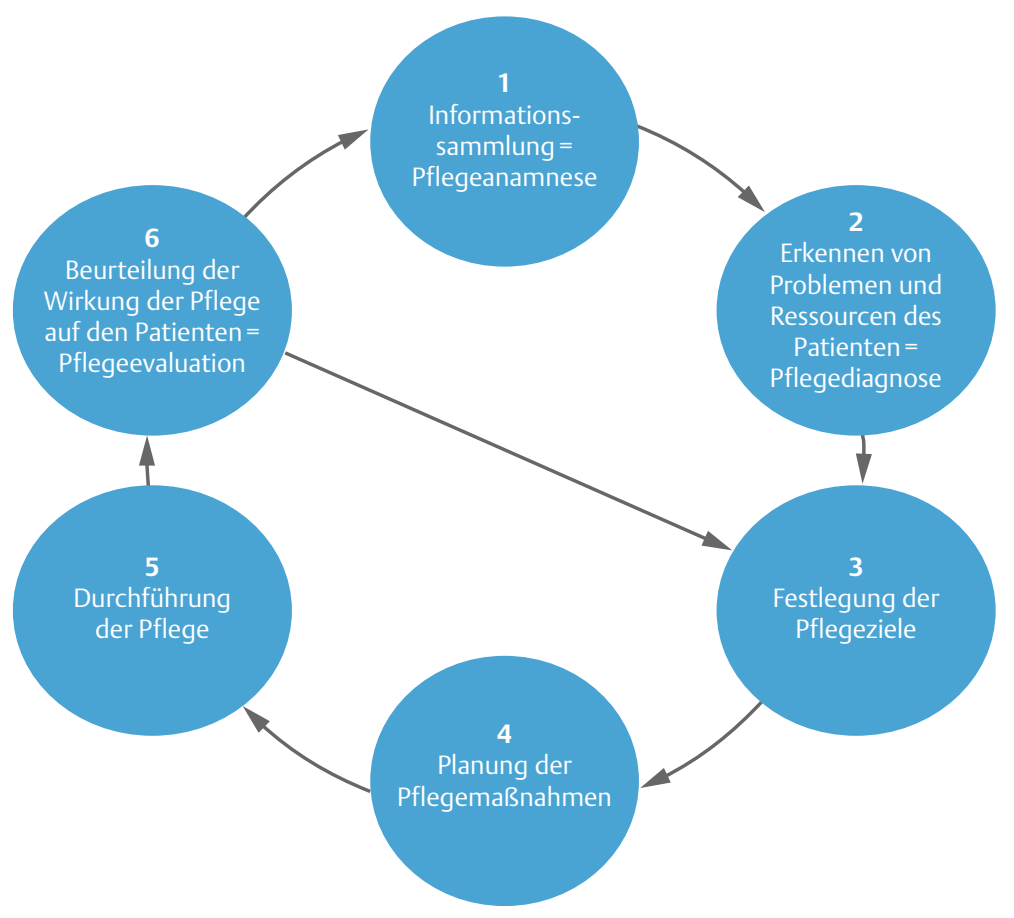

Abb. 1 Pflegeprozessmodell. Modifiziertes 6-Phasen-Modell nach Fiechter und Meier (1998).

regelmäßig von seinen zwei Enkelkindern besucht. Vor der Berentung arbeitete Herr N. als technischer Angestellter. Zu Hause hört er gerne Schlagermusik und verbringt viel Zeit am Computer. Bisher war er selbstversorgend. Eine privat finanzierte Haushaltshilfe erledigte zweimal in der Woche den Einkauf, den Haushalt und die Wäsche. Täglich erhielt der Patient fertig zubereitetes Essen, das ebenfalls privat finanziert wurde. Bislang konnte Herr N. seine Geldgeschäfte selbstständig erledigen.

\section{Pflegeproblem „Sich bewegen“ nach dem Pflegeprozessmodell nach Fiechter und Meier \\ Probleme}

Die Bewegung ist aufgrund der Hüftgelenksendoprothese rechts, der damit verbundenen Schmerzen und des allgemeinen Kraftverlusts stark eingeschränkt. Sensibilitätsstörungen werden durch die vorhandene Polyneuropathie mit einem Kribbeln und Missempfindungen gegenüber Hitze-, Kälte- und Schwellungsgefühlen angegeben. Infolgedessen ging das Bewegungsund Haltungsgefühl verloren. Aufgrund der Herzinsuffizienz haben sich in beiden Beinen Unterschenkelödeme ausgebildet.

Der Positionswechsel im Bett von der Rückenlage in den Sitz erfolgt mit wenig Hilfe, aber unter enormem Krafteinsatz. Die Transfers Sitzen-Sitzen und Sitzen-Stehen sowie Stehen und Gehen sind nur mit umfangreicher Unterstützung einer Pflegefachkraft möglich. Dies entspricht der Bedarfsgruppe 3 der Aktivierend-therapeutischen Pflege in der Geriatrie (ATP-G). Laut Esslinger Transferskala ist nach dem Akutereignis geschulte Laienhilfe erforderlich (Hilfestufe 2). Der Patient ist in seiner Aufmerksamkeit und Konzentration herabgesetzt und damit stark sturzgefährdet (Assessment nach S. Huhn bei Aufnahme 19 von 24 Punkten).

\section{Ressourcen}

Herr N. verfügt über ausreichende Kopfund Rumpfstabilität. Mikrobewegungen im Bett werden eigenständig durchgeführt, dabei ist die linke, gesunde Seite in der Bewegung nicht eingeschränkt. Das stabile Gleichgewicht kann mit dem Einsatz des hohen Gehwagens gehalten werden und im Rollstuhl ist die Fortbewegung selbstständig. Auftretende Schmerzen im rechten Oberschenkel werden auf Nachfrage mittels NRS (Numerische Ratingskala) mit 5 von 10 angegeben. Der Patient ist motiviert und möchte schnell wieder in seiner Mobilität eigenständig werden.

Ängste vor einem erneuten Sturzereignis werden nicht angegeben. In der Häus-

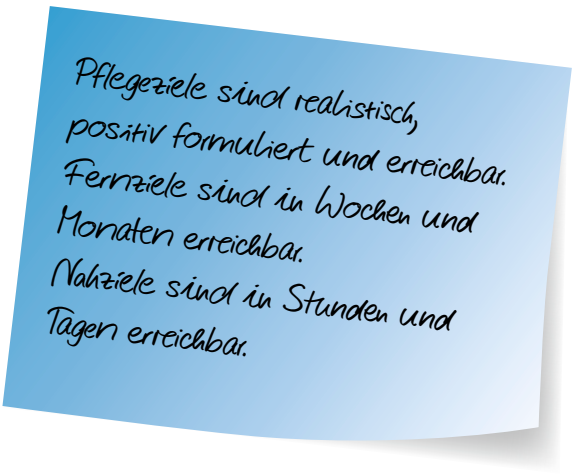

lichkeit sind Haltegriffe im Badezimmer und ein Notrufknopf bereits vorhanden. Momentan ist eine Gehstrecke mit einem hohen Gehwagen unter Vollbelastung von 50 Metern mit einer Pause möglich.

\section{Pflegeziele}

Das gemeinsame Fernziel des Patienten und des therapeutischen Teams ist eine selbstständige, sichere und koordinierte Bewegung mit individuell angepasstem Hilfsmittel in und außerhalb der Häuslichkeit. Nahziele sind die Erhaltung und Förderung der vorhandenen Ressourcen. Der Patient nimmt aktiv beim Kraft-, Balance-, Ausdauer- und Aufstehtraining teil. Er nimmt Veränderungen in seiner Aufmerksamkeit und Konzentration bewusst wahr. Aufgrund einer bereits angepassten Schmerzmedikation wurden die Schmerzen besser. Die Körperwahrnehmung und das Gleichgewicht sind verbessert.

Der Positionswechsel im Bett von der Rückenlage in den Sitz erfolgt mit weniger Kraftanstrengung und der tiefe Transfer sowie der Transfer über den Stand erfolgen mit Unterstützung und Anleitung der Pflegefachkraft. Eine Gehstrecke mit dem Rollator von 100 Metern wird angestrebt. Es erfolgt eine Beratung zur Sturzprophylaxe und Risikofaktoren werden besprochen. Der Rollator wird als Hilfsmittel zur Unterstützung von Bewegungsübergängen außerhalb des Bettes angepasst und es erfolgt eine Anleitung dazu.

\section{Pflegemaßnahmen}

Der Patient wird täglich auf Veränderungen in der Gemütslage, der Motivation, der Muskelkraft, des Gleichgewichts, der Ausdauer, der Orientierung, der Konzentration, der Sensibilitätsstörungen und des Hautzustands beobachtet. Alle ausgewählten Maßnahmen zur Bewegungsförderung werden nach Prinzipien aus dem BobathKonzept, der basalen Stimulation und der Aktivierend-therapeutischen Pflege im 
Umsetzen nach den Prinzipien des Bobath-Konzepts und der Aktivierend-therapeutischen Pflege in der Geriatrie

Das Bobath-Konzept ist ein therapeutisch aktivierendes Konzept, das sich in ständiger Weiterentwicklung befindet. Es ist keine Methode, sondern es wird nach Prinzipien gearbeitet. Die Anwendung ist nicht nur für den klassischen Schlaganfallpatienten vorbehalten. Fundamente des Bobath-Konzepts sind die Förderung der Aktivierung des Patienten für eine bessere Haltungskontrolle und eine bessere Körperwahrnehmung während jeder pflegerischen Aktivität des täglichen Lebens.
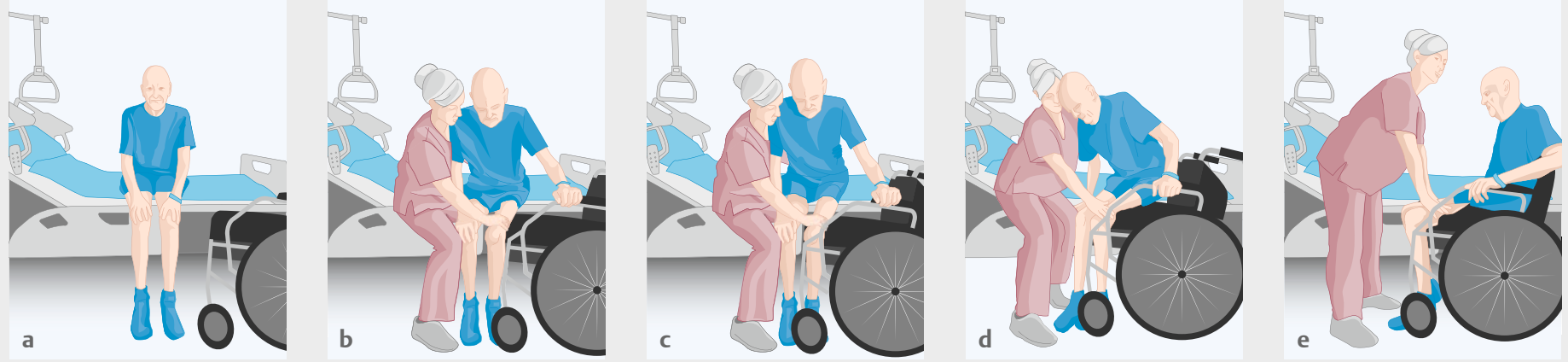

Abb. 2 Tiefer Transfer in den Rollstuhl mit seitlicher Unterstützung. a Die Matratze bietet eine stabile Unterstützungsfläche, beide Füße stehen fest auf dem Boden. b Transfer mit seitlicher Unterstützung der Pflegekraft, Oberkörper des Patienten kommt nach vorne, die linke Hand wird durch das Halten an der Rollstuhllehne zur Orientierung genutzt. c Das Becken richtet sich auf und das Gewicht wird nach vorne auf die Füße gebracht. d Das Gesäß wird zur Seite versetzt, die Pflegekraft kontrolliert die Stellung der Beine zueinander. e Endposition: stabiler Sitz im Rollstuhl.

USF (Unterstützungsfläche): Die Größe der angenommenen Unterstützungsfläche beeinflusst den Muskeltonus. Für Aktivität muss die USF stabil sein, um sie gut nutzen zu können.

Muskeltonus: Der Tonus muss hoch genug sein, um gegen die Schwerkraft bestehen zu können, gleichzeitig muss er niedrig genug sein, um Bewegungen zuzulassen. Es gilt: Stabilität vor Mobilität!

Schlüsselpunkte: Die Stellung der Schlüsselpunkte zueinander leitet Bewegung ein. Es gibt zentrale, proximale und distale Schlüsselpunkte. Für Bewegungen sollten die proximalen Schlüsselpunkte näher zum zentralen Schlüsselpunkt liegen.

therapeutischen Team ausgewählt und umgesetzt.

Um die Motivation zu fördern, werden alle Schritte mit dem Patienten ausgewählt, erläutert und demonstriert. Eine somatische Stimulation des Körpers, insbesondere der Füße bei einer bestehenden Polyneuropathie, begünstigt die Körperwahrnehmung und bereitet den Patienten auf folgende Bewegungsabläufe vor. Eine Schmerzerfassung erfolgt regelmäßig mindestens vor jeder Mobilisation mittels NRS.

Um schnellstmögliche Erfolge zu verzeichnen, werden zunächst einfach umzusetzende Techniken zum besseren Transfer im Bett von der Rückenlage in den stabilen Sitz auf die Bettkante gezeigt. In der liegenden Grundposition im Bett stehen die proximalen Schlüsselpunkte deutlich näher zum Zentrum und es wird ausreichende Unterstützungsfläche angeboten, um den Muskeltonus zu normalisieren. Die Beine werden eigenständig aufgestellt und der Patient wird zur Rotation auf die linke Körperseite aufgefordert. Der Bewegungsübergang zur weniger betroffenen Seite ist aufgrund der Funktionseinschränkung be- wusst ausgewählt. Die Knie werden so nah wie möglich an den Körperstamm gebracht und die Füße über dem Bettende positioniert. Durch die Anspannung der Rumpfmuskulatur stützt sich der Patient über den abgelegten Unterarm auf dem Bett sitzend in Richtung Bettkante.

Patientenbezogene Sturzrisikofaktoren, wie die Aufmerksamkeitsminderung, Sensibilitätsstörungen in den Füßen und bereits durchgemachte Stürze in der Vorgeschichte, werden vom Patienten erkannt und angepasst. Auch medikamenten- und umgebungsbezogene Risikofaktoren, wie der Einsatz von Opiaten sowie Umgebungsfaktoren (Beleuchtung, Boden, Platzangebot im Zimmer, passendes Schuhwerk), sind dabei zu berücksichtigen. Eine weitere Maßnahme zur Bewegungsförderung ist die Anleitung zum tiefen Transfer in den Rollstuhl ( $\rightarrow$ Abb. 2).

Da Herr N. schon nach 2 Tagen mit dem hohen Gehwagen stabil und sicher mobil war, erfolgt nun nach einer Hilfsmittelanpassung die Mobilisation mit dem Rollator.

Für die erreichbaren Ziele werden dem Patienten genügend Zeit und Raum gegeben.

\section{Zur Förderung und Erhaltung der Motivation und Aufmerksamkeit werden Pflegehandlungen und Gespräche empathisch und durch aktives zuhören gestal- tet.}

Um eine einheitliche und nachvollziehbare Herangehensweise für alle beteiligten Berufsgruppen nachzuweisen, werden alle durchgeführten Maßnahmen dokumentiert und bei der täglichen Frühbesprechung, der Visite sowie der wöchentlichen Teamsitzung ausgetauscht. Pflegeziele werden neu formuliert und angepasst.

Alle Bewegungsübungen werden über den Tag verteilt und mit anderen pflegerischen Handlings und Selbsthilfetraining, z. B. bei der täglichen Körperpflege, Begleitung zur Toilette und Nahrungsaufnahme am Tisch, verknüpft. Dies beugt zusätzlich einem Dekubitus und einer Pneumonie vor. 
Expertenstandard "Sturzprophylaxe"
über: https: / / ww dingp.de

Eine ausführliche Beratung zur Sturzprävention, geleitet nach dem aktuellen Expertenstandard in der Pflege, erfolgt in den nächsten Tagen. Hierbei werden Informationen für die Anpassung von Hilfsmitteln, der häuslichen Umgebungsgestaltung und einer vollwertigen Ernährung vermittelt.

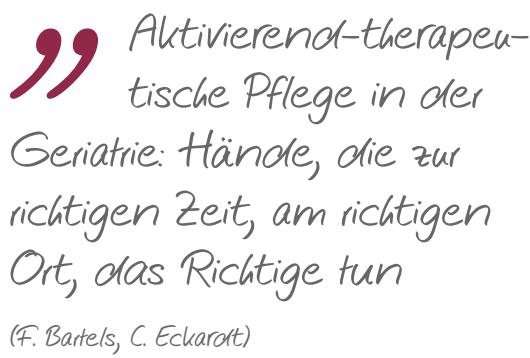

Evaluation

In erster Linie standen die Förderung der Selbstständigkeit, die Steigerung der Aufmerksamkeit und der Erhalt der Motivation zur individuell definierten Lebensqualität im Vordergrund. Hierbei wurden vorhandene Fähigkeiten und Fertigkeiten beachtet sowie kleinste Fortschritte und Erfolge zur weiteren Motivation und Krankheitsbewältigung positiv bekräftigt.

Zum Zeitpunkt der Entlassung war der Patient in der Lage, selbstständig den Transfer aus der Rückenlage im Bett in eine aufrechte und stabile Sitzposition ohne große Kraftanstrengungen durchzuführen. Das Gehen mit dem Rollator bedarf noch der Begleitung. Hier besteht weiterhin ein hohes Sturzrisiko, da der Patient nicht immer aufmerksam und konzentriert bei den Transferübungen ist. Dabei überspielt er unkontrollierte Bewegungsabläufe. Infolgedessen ist weiterhin situativ eine wiederholte Beratung zur Sturzprävention notwendig.

Bestehende Ressourcen, wie Motivation und ein stabiles Gleichgewicht, konnten erhalten und gefördert werden. Die Wegstrecke mit dem Rollator wurde auf 100 Meter erweitert. Der Sozialdienst veranlasste den Pflegegrad 2 sowie eine weitere Behandlung für 4 Wochen in der Kurzzeitpflege. Der Schlaf- und Wohnbereich seines Einfa-

Tab. 1 Übersicht über die durchgeführten Assessments.

\begin{tabular}{|c|c|c|c|c|}
\hline Bereich & Assessment & Aufnahme & Entlassung & Resultat zur Entlassung \\
\hline $\begin{array}{l}\text { Körperliche Selbstver- } \\
\text { sorgungsfähigkeiten }\end{array}$ & Barthel-Index & $40 / 100$ & $65 / 100$ & hilfsbedürftig \\
\hline ATP-G Bedarfsgruppen & & $\begin{array}{l}\text { Bedarfs- } \\
\text { gruppe } 3\end{array}$ & $\begin{array}{l}\text { Bedarfs- } \\
\text { gruppe } 1\end{array}$ & $\begin{array}{l}\text { geringfügige Hilfe- } \\
\text { stellung erforderlich }\end{array}$ \\
\hline Mobilität & $\begin{array}{l}\text { Esslinger } \\
\text { Transferskala }\end{array}$ & Hilfestufe 2 & Hilfestufe 1 & $\begin{array}{l}\text { spontane, ungeschulte } \\
\text { Laienhilfe erforderlich }\end{array}$ \\
\hline Kognition & $\begin{array}{l}\text { Mini-Mental } \\
\text { Status }\end{array}$ & $24 / 30$ & $26 / 30$ & $\begin{array}{l}\text { keine oder leichte Ein- } \\
\text { schränkung }\end{array}$ \\
\hline Kognition & Uhrentest & keine Fehler & keine Fehler & $\begin{array}{l}\text { komplexe Handlungs- } \\
\text { planung und visuell- } \\
\text { konstruktive Fähigkeiten }\end{array}$ \\
\hline Emotion & $\begin{array}{l}\text { Geriatrische } \\
\text { Depressionsskala }\end{array}$ & 2 & 3 & unauffällig \\
\hline Ernährung & $\begin{array}{l}\text { Mini Nutritional } \\
\text { Assessment }\end{array}$ & $21 / 30$ & $22 / 30$ & $\begin{array}{l}\text { Risikobereich für } \\
\text { Mangelernährung }\end{array}$ \\
\hline $\begin{array}{l}\text { Instrumentelle Selbst- } \\
\text { versorgung }\end{array}$ & IADL & $2 / 5$ & $2 / 5$ & $\begin{array}{l}\text { eingeschränkte } \\
\text { Fähigkeiten }\end{array}$ \\
\hline Schmerzen & $\begin{array}{l}\text { Numerische } \\
\text { Ratingskala }\end{array}$ & $5 / 10$ & $3 / 10$ & gut \\
\hline Sturzrisiko & S. Huhn & $19 / 24$ & $14 / 24$ & sehr hohes Risiko \\
\hline
\end{tabular}

milienhauses inkl. Computer wurden bereits nach einem Beratungsgespräch mit dem Sohn in die untere Etage verlegt.

Durch einen Pflegedienst wird die Pflege der unteren Extremitäten, die Kompressionstherapie, „Essen auf Rädern“ und das Stellen der Medikamente gewährleistet, da die IADL (instrumentelle Alltagsaktivitäten) sich gegenüber der Aufnahme nicht verändert hatten.

Insgesamt können die für den Patienten bisher erreichten Ziele als erfolgreich bewertet werden. Diese konnten aufgrund einer guten interdisziplinären $\mathrm{Zu}$ sammenarbeit, der standardisierten Assessments ( $\rightarrow$ Tab. 1), der professionellen Anwendung aktueller Pflegekonzepte und der fachlich geschulten Pflegefachkräfte erreicht werden. Der Patient wurde mit der Bedarfsgruppe 1 der Aktivierend-therapeutischen Pflege in der Geriatrie entlassen.

\section{Literatur}

1 Bartels F, Eckardt C, Wittekindt S, Wittrich A, Hrsg. Aktivierend-therapeutische Pflege in der Geriatrie, Band 1: Grundlagen und Formulierungshilfen. Stuttgart: Kohlhammer; 2015
2 Böhmer F, Füsgen I, Hrsg. Geriatrie. Der ältere Patient mit seinen Besonderheiten, Wien/Köln/Weimar: Böhlau; 2008

3 Friedhoff M, Schieberle D, Hrsg. Praxis des Bobath-Konzeptes, Grundlagen - Handling - Fallbeispiele. Stuttgart: Thieme; 2014

4 Hatz-Casparis M, Roth-Sigrist M, Hrsg. Basale Stimulation ${ }^{\circledR}$ in der Akutpflege. Handbuch für die Pflegepraxis. Bern: Hans Huber; 2012

5 Hochschule Osnabrück, Hrsg. Expertenstandard Sturzprophylaxe in der Pflege, http://www.dnqp. de/fileadmin/groups/607/Sturz-Akt_Auszug.pdf, Zugriff: 01.07.2015

6 I care Pflege, Stuttgart: Thieme; 2015

7 Schuler M, Oster P, Hrsg. Geriatrie von A bis Z der Praxisleitfaden. Stuttgart: Schattauer; 2008

\section{Autor}

René Thiel

Gesundheits- und Krankenpfleger, übergeordneter Praxisanleiter, Fachpflegekraft Aktivierend-therapeutische Pflege Geriatrie, Wundexperte ICM e. V.,

Algesiologische Fachassistenz, Peer Tutor Kinaesthetics

rene.thiel@uksh.de

\section{Bibliografie}

DOI 10.1055/s-0043-117777

GGP 2017; 1: 104-107

(C) Georg Thieme Verlag KC

Stuttgart · New York · ISSN 2511-7548 